\title{
Concrete Strategy of Constructing the Ecological Environment of Regional Science and Technology Finance
}

\author{
Fengxiang Jiang \\ Xi’an Peihua University, Xi’an Shaanxi, 710125
}

Keywords: Region; science and technology finance; ecological environment; strategy

\begin{abstract}
The combination of science and technology with finance plays a great role in promoting regional economic growth and perfecting the theoretical system of the development of science and technology finance to a certain extent. At the same time, the S \& T financial system has become a necessary condition and an important guarantee for the implementation of S \& T strategy. The value orientation of $S \& T$ finance-S \& $T$ enterprises are the main absorption force of emerging technologies. Only the coordinated development of science and technology and finance can realize the development of regional economy and provide real capital service for the real economy and industrial development in the development of regional economy.
\end{abstract}

\section{Introduction}

Science and technology finance began its research in 1987, which hits its hottest level in 2015. In the report of the Nineteenth National Congress of China, the regional cooperative development strategy is proposed, and the research on the regional science and technology finance cooperative development strategy is strengthened to realize the regional coordinated development. The relevant literature mainly focuses on the dynamic mechanism of the regional science and technology finance development. The bottleneck factors of the development of regional science and technology finance, the regional science and technology innovation and the regional science and technology finance coordinated development and so on. Xu Yulian and Wang Yudong (2013) put forward that regional scientific and technological innovation needs the support of science and technology finance, and the development of regional science and technology finance also needs the support of science and technology innovation. Zhong Shuqin, Li Yan, Liu Fan, et al. (2016) put forward that in terms of regional scientific and technological innovation work, its rapid advancement is supported by science and technology finance, and the development of science and technology finance also needs the support of science and technology innovation. Only by supporting each other can the coordinated development of science and technology finance and regional science and technology innovation be realized. Liu Yunling (2018) pointed out that there are some problems in the development of science and technology finance, such as the single financing mode, the lack of linkage mechanism, the lag of the development of public credit information platform, and so on, which seriously restrict the development of science and technology finance in China. In view of this situation, government management departments at all levels of our country have also actively promoted the establishment of a scientific, technological and financial development guarantee mechanism in the areas of policies, regulations, relevant theories, practical research, and so on, through the exertion of their own leading role. In order to effectively promote the realization of sustainable development goal of science and technology finance in China. Wang Xinlei and Zeng Yu (2018) put forward that in the cooperative development of Beijing-Tianjin-Hebei, there are some problems and reasons for the slow sharing of scientific and technological financial resources, the looseness of economic ties and the sharing of human resources, and the improvement of the sharing system of scientific and technological financial resources. The information innovation platform system and the talent incentive system are put forward the corresponding countermeasures and suggestions. Lu Yajuan and Liu Hua (2018) proposed that scientific and technological innovation should be guaranteed by financial innovation and the steady development of finance should be supported by scientific and technological innovation and emerging industries. The organic 
combination of science and technology and finance plays an important role in promoting regional economic growth.

\section{The Connotation of Science and Technology Financial Environment}

At present, domestic scholars' research on the ecological environment of science and technology finance is still blank, but science and technology finance is also an important part of the financial ecological environment, derived from ecology, is also a bionic concept, the financial and ecological environment must be analyzed by means of financial ecology. Professor Wen Yuechun used ecological engineering methods to study the technological innovation investment and financing financial ecology as the core circle, the external environment circle and the internal environment circle. At the same time, the three main bodies of technology enterprises, government and financial institutions are taken as the core circle; the internal environment circle is composed of five essential elements: policy, technology, talent, capital and resources; the external environment circle is mainly the intermediary service system, credit system and law. The system is composed of the environment. This paper thinks that Professor Wen Yuechun's research perspective is worthy of reference. When analyzing the scientific and technological financial ecological environment, he starts from the perspective of financial ecological environment but should consider the characteristics of science and technology finance. Zhao Changwen's description of the technology financial environment in the book Science and Technology Finance is that the technology financial environment is science and technology finance. Legal, economic, cultural, and social systems, institutional and traditional environments in which financial instruments (technical loans, venture capital investments, technology insurance, financial technology inputs, and technology capital markets) operate mainly include credit systems, guarantee systems, contractual awareness, entrepreneurship and legal systems and government policies.

This paper attempts to define the ecological environment of science and technology finance, and holds that the ecological environment of science and technology finance is the external environment which takes science and technology finance as the main body and influences and restricts the development of science and technology finance, including the legal environment, the credit environment and the government environment. Sustainable development ability, citizen and enterprise contract, etc.

\section{Specific Strategies for Building a Regional Science and Technology Financial Ecological Environment}

\subsection{Economic Environment}

\subsubsection{To Change Development Ideas and Optimize Industrial Structure}

From the point of view of economic environment, there are some problems such as unbalanced development of industrial structure in China, which seriously restricts the sustainable growth of economy. Therefore, we should speed up the transformation of the thinking of industrial development, promote the development of secondary and tertiary industries, and further optimize the allocation of resources, so as to promote the development of industries to capital-intensive and technology-intensive high-tech industries.

\subsubsection{To Build a Service Platform and Integrate Social Resources}

In order to better promote the coupling of regional science and technology and finance, we must pay attention to the integration of regional science and technology financial resources and build a public service platform to solve the problem of information asymmetry between science and technology enterprises and financial institutions. Under the guidance of the government, the development environment of scientific and technological market oriented and social intermediary service institutions should be established, which aims at providing more financing guarantee, venture capital and listed financial services for science and technology enterprises. At the same time, 
it can effectively integrate science and technology and financial resources, promote the participation of banks, insurance, venture capital, guarantee and other financial institutions with scientific and technological enterprises, form a resource sharing information database, and classify and evaluate the financing needs of science and technology enterprises. At the same time, we should perfect the capital market system, promote the standard development of indirect financing, actively develop direct financing, increase the proportion of direct financing, and support the rapid growth of high-tech enterprises. Actively promoting the development of various types of science and technology financial innovation subjects, such as speeding up the development and rational distribution of science and technology microfinance companies, actively exploring financing methods that combine loans with investment, and so on: attaching importance to giving full play to venture capital, the function of high-tech small and medium-sized enterprises incubator of financial institutions such as venture capital and the establishment of non-listed enterprise equity trading mechanism not only provide investment opportunities for venture capital funds but also clear the exit channels of investors to relieve their worries.

\subsection{Financial Environment Optimization}

\subsubsection{To Increase Investment in Science and Technology Finance and Promote the Development of Technology Banks}

The financial support for science and technology finance is mainly reflected in the financial expenditures of financial technology. Technology finance in the early stage of development, the government needs to support it and promote it through financial subsidies in the form of loan risk quasi-funds. Financial institutions finance technology-based companies. Financial technology withdrawals also involve investment in $R \& D$ funding. $R \& D$ order the investment in the segment is conducive to the accumulation of science and technology, reserve for technological innovation, and achieve a benign approach to science and technology finance. Ring development. From the analysis of the financial environment, the investment in science and technology finance is the ecological and financial environment of Hunan Province. The overall level of development is crucial. In order to optimize the ecological environment of science and technology finance, we should vigorously develop the technology bank as the generation. The technology financial institutions of the table realize the efficient connection between the technology-based technology financing needs and the technology banks. There is a gradual reform of the financing model to adapt to the development of science and technology finance. High-risk, high-yield for technology-based companies the characteristics of the multi-level financing capital market should also be established and improved.

\subsubsection{Perfecting the Venture Capital Market and Developing the Financing Channel}

At present, the venture capital, angel investment and private venture capital in various regions are still weak, the multi-level venture capital market still needs to be established, and this cannot meet the financing needs of high-tech enterprises in each stage of development. According to the characteristics of venture capital, the corresponding mechanism should be established, and the government should take the initiative to guide, including the establishment of a perfect enterprise credit bank, the establishment of an information platform for investment and financing, the improvement of the exit mechanism and the legal environment, etc. So as to promote the docking between high-tech enterprises and venture capital institutions, you can learn from the Suzhou Municipal Venture Capital Fund launched the same share, professional operation model. It is also possible to establish venture capital funds by effectively guiding private capital to finance high-tech enterprises.

\subsection{To Optimize Social Environment}

\subsubsection{To Establish an Innovation-driven Strategy}

The establishment of innovation-driven development strategy system is helpful to promote the implementation of innovation-driven development strategy. Through the in-depth study of the factors related to the innovation-driven development strategy, the comprehensive integration of 
existing resources in science and technology, finance and human resources, as well as the supplement of the preferential policies and measures for innovation-driven strategies, the establishment and improvement of innovation drive development strategy system. It is suggested that in the construction of innovation-driven development strategy system, science and technology finance should be established as an important component, and the existing policies and systems of science and technology finance should be revised and perfected. The perfection of science and technology finance will promote its own development, and the development of science and technology finance will have the power to push forward the implementation of innovation-driven development strategy.

\subsubsection{To Improve the Policy System}

Like other finances, technology finance is a social system project that requires a sound legal system to protect it, thus making the technology finance operation legally compliant. At present, there are relatively few laws and regulations on science and technology in various regions. Although the existing policies have promoted the development of science and technology finance to a certain extent, there are still many shortcomings in these measures from a global perspective, and it is necessary to further improve the relevant rules. The government has formulated science and finance support policies, strengthened overall planning and top-level design, actively exerted the leverage effect of fiscal funds, and encouraged institutions such as finance, securities, insurance, and venture capital to intensify financial innovation and promote the two-way integration of technological innovation and financial capital. The technology enterprises in the growth stage provide personalized and professional services; strengthen the innovation of science and technology financial service organizations, establish a policy-based financial support system for small and medium-sized enterprises; explore the establishment of financial service centers for technology enterprises, and innovate service concepts and models to provide corporate financing One-stop service; strengthen the construction of social credit system and optimize the innovation and entrepreneurship environment of science and technology enterprises.

\subsection{To Create Sustainable Development Environment $\square$}

\subsubsection{To Cultivate a Good Atmosphere for Technological Innovation}

A good atmosphere for technological innovation means not only the recognition of science and technology innovation by society, but also the failure of society. Technological innovation stems from people's reality and potential needs, these needs it is often achieved through the efforts of scientific researchers, and part of it is through people's pursuit of their own ideas. In order to achieve this, there will be different degrees of setbacks in this implementation process; they need social recognition and support. Therefore, we should vigorously promote the spirit of innovation in the society and create a good social environment for self-employment. The government should add strong $R \& D$ expenditures are encouraged to encourage enterprises to invest in scientific research and provide preferential tax policies.

\subsubsection{To Cultivating Talents for Scientific and Technological Innovation}

Innovation-driven development strategy is the core of science and technology innovation, its key is to train all kinds of talents needed by society. In the process of construction of science and technology financial system, talents are needed in intangible asset collateral assessment, venture capital management, guarantee and so on. This puts forward new requirements to the social education system, such as retaining students' innovative thinking, encouraging their innovative ideas, and cultivating compound talents. Through the short-term training courses to the existing relevant industry personnel related knowledge training, re-education and short-term charging. 


\section{Conclusion}

In short, the development of science and technology finance needs to continuously optimize its development environment, including economic environment, political environment, financial environment, social environment, sustainable development environment, and so on. It is necessary to speed up scientific and technological innovation in financial enterprises and build an efficient multi-level service quality system.

\section{Acknowledgement}

Fund Project: This article was funded by Xi'an Peihua College-level Scientific Research Project. Project Name: Regional Science and Technology Finance Collaborative Development Strategy Research; Project No.: PHKT18051

\section{References}

[1] Wang Xinlei, Zeng Yi. Research on the Innovation of Science and Technology Financial System Coordinated by Beijing, Tianjin and Hebei [J].Financial Economy, 2018(6):77-79

[2] Jin Yufang. Research on the Status Quo and Innovation Strategy of Science and Technology Finance Based on Commercial Banks [J].Trade in Economics, 2018(4):148-149

[3] Liu Yuling. Research on the Development of Science and Technology Finance in China [J]. Taxation, 2018(5): 174+177

[4] Lu Yajuan, Liu Wei. Analysis of the Correlation Effect between Synergic Agglomeration of Science and Technology Finance and Regional Economic Growth [J]. Research on Financial and Economic Issues, 2018(2): 64-70.

[5] Zhang Yuhua, Zhang Tao. Research on the Impact of Science and Technology Finance on the Cooperative Agglomeration of Producer Service Industry and Manufacturing Industry [J]. China Soft Science, 2018(3): 47-55

[6] Zhong Shuqin, Li Yanzhong, Liu Fan, et al. Analysis of the coordinated development model of regional science and technology innovation and science and technology finance [J]., 2017, 20(20): 165-165.

\footnotetext{
About the Author:

Fengxiang Jiang (1983- ), female, Han nationality, Hunan Xiangyin, associate professor of accounting and finance college, Xi'an Peihua College, research direction: human resource management and regional economics.
} 